\title{
Uma Extensão do Teorema de Seleção de Filippov para Escalas Temporais
}

\author{
Iguer Luis D. dos Santos \\ Departamento de Matemática, FEIS, UNESP, \\ 15385-000, Ilha Solteira, SP \\ E-mail: iguerluis@mat.feis.unesp.br.
}

Resumo: Neste trabalho, obtemos uma generalização de um resultado clássico de seleção mensurável útil na teoria do controle ótimo. Assim, obtemos uma extensão do teorema de seleção de Filippov para escalas temporais.

Palavras-chave: Seleção Mensurável de Filippov, Inclusões Dinâmicas, Escalas Temporais

\section{Introdução}

Obtemos uma generalização do teorema de seleção de Filippov. Assim, obtemos uma seleção $\Delta$-mensurável do campo vetorial da inclusão dinâmica associada com os sistemas de controle de equações estudados, exigindo as hipóteses usuais do caso contínuo [5]. A classe de sistemas de controle de equações considerada aqui foi abordada na literatura [7] para o estudo de condições necessárias a otimalidade. Diferente de [7], as trajetórias de estado para os sistemas de controle estudados são arcos e as funções de controle delta mensuráveis.

Outras extensões do teorema de seleção de Filippov para escalas temporais podem ser encontradas em [12] e [13].

\section{Preliminares}

Nessa seção consideramos conceitos e resultados básicos que serão utilizados ao longo do trabalho.

\subsection{Escala temporal}

Uma escala temporal $\mathbb{T} \subset \mathbb{R}$ é um conjunto não-vazio e fechado. Usaremos uma escala temporal $\mathbb{T}$ compacta, sendo $a=\min \mathbb{T}$ e $b=\max \mathbb{T}$.

Se $A$ é um subconjunto de $\mathbb{R}$, denotamos por $A_{\mathbb{T}}$ o conjunto $A \cap \mathbb{T}$.

Define-se a função $\sigma: \mathbb{T} \rightarrow \mathbb{T}$ como

$$
\sigma(t)=\inf \{s \in \mathbb{T}: s>t\}
$$

e a função $\rho: \mathbb{T} \rightarrow \mathbb{T}$ como

$$
\rho(t)=\sup \{s \in \mathbb{T}: s<t\} .
$$

Estamos supondo que $\inf \emptyset=\sup \mathbb{T}$ e $\sup \emptyset=\inf \mathbb{T}$.

Lema 2.1 ([3]). Existem $I \subset \mathbb{N} e\left\{t_{i}\right\}_{i \in I} \subset \mathbb{T}$ tal que

$$
R S:=\{t \in \mathbb{T}: t<\sigma(t)\}=\left\{t_{i}\right\}_{i \in I} .
$$


Defina $\mathbb{T}^{\kappa}:=\mathbb{T} \backslash(\rho(\sup \mathbb{T}) \text {, sup } \mathbb{T}]_{\mathbb{T}}$. Considere uma função $f: \mathbb{T} \rightarrow \mathbb{R}$ e $t \in \mathbb{T}^{\kappa}$. Se existe $\xi \in \mathbb{R}$ tal que, para todo $\varepsilon>0$ existe $\delta>0$ de modo que

$$
|f(\sigma(t))-f(s)-\xi(\sigma(t)-s)| \leq \varepsilon|\sigma(t)-s|
$$

para todo $s \in(t-\delta, t+\delta)_{\mathbb{T}}$, diz-se que $\xi$ é a derivada delta de $f$ em $t$ e denota-se $\xi=f^{\Delta}(t)$.

Dada uma função $f: \mathbb{T} \rightarrow \mathbb{R}^{n}$ e $t \in \mathbb{T}^{\kappa}$, diz-se que $f$ é $\Delta$-diferenciável em $t$ se cada função coordenada $f_{i}: \mathbb{T} \rightarrow \mathbb{R}$ for $\Delta$-diferenciável em $t$. Neste caso $f^{\Delta}(t)=\left(f_{1}^{\Delta}(t), \ldots, f_{n}^{\Delta}(t)\right)$.

\subsection{Integração em escalas temporais}

A obtenção da $\sigma$-álgebra de subconjuntos da escala temporal $\mathbb{T}$, constituída de conjuntos $\Delta$ mensuráveis, pode ser encontrada em [6]. Denotaremos essa $\sigma$-álgebra por $\Delta$ e a $\Delta$-medida de Lebesgue por $\mu_{\Delta}$.

Lema $2.2([3])$. Tome $E \subset \mathbb{T}$. Então $E$ é $\Delta$-mensurável se, e somente se, E é Lebesgue mensurável.

Para funções $f: \mathbb{T} \rightarrow \overline{\mathbb{R}}$ a noção de integração pode ser encontrada, por exemplo, em [1], [9] e [11]. Denotamos a integral de uma função $f: \mathbb{T} \rightarrow \overline{\mathbb{R}}$ sobre um conjunto $E \in \Delta$ por

$$
\int_{E} f(s) \Delta s
$$

Chamamos essa integral de $\Delta$-integral de Lebesgue de $f$ sobre $E$. Denotaremos por $L_{1}\left(E, \mathbb{R}^{n}\right)$ o conjunto das funções $f: \mathbb{T} \rightarrow \mathbb{R}^{n} \Delta$-integráveis sobre $E$.

Dada uma função $f: \mathbb{T} \rightarrow \mathbb{R}^{n}$ defina $\tilde{f}:[a, b] \rightarrow \mathbb{R}^{n}$ por

$$
\tilde{f}(t)=\left\{\begin{array}{l}
f(t), t \in \mathbb{T} \\
f\left(t_{i}\right), \quad t \in\left(t_{i}, \sigma\left(t_{i}\right)\right) \text { para algum } i \in I
\end{array}\right.
$$

onde $I \subset \mathbb{N}$ e $\left\{t_{i}\right\}_{i \in I}=R S$.

O resultado a seguir é provado em [3] para funções escalares. Entretanto, esse resultado continua válido para funções vetoriais, como afirmado a seguir.

Proposição 2.1 ([3]). Tome uma função $f: \mathbb{T} \rightarrow \mathbb{R}^{n}$. Então $f$ é $\Delta$-mensurável se, e somente se, $\tilde{f}$ é $\mathcal{L}$-mensurável.

Lema 2.3 ([12]). Seja $u: \mathbb{T} \rightarrow \mathbb{R}^{m}$ uma função $\Delta$-mensurável. Se A é Borel mensurável então $u^{-1}(A) \in \Delta$.

Como a função $\sigma: \mathbb{T} \rightarrow \mathbb{T}$ é $\Delta$-mensurável [6] temos o seguinte corolário.

Corolário 2.1. Se $x: \mathbb{T} \rightarrow \mathbb{R}^{n}$ é uma função contínua então a função $x \circ \sigma: \mathbb{T} \rightarrow \mathbb{R}^{n}$ é $\Delta$-mensurável.

\subsection{Funções absolutamente contínuas em escalas temporais}

Definição 2.1. Diz-se que uma função $f: \mathbb{T} \rightarrow \mathbb{R}^{n}$ é absolutamente contínua se para todo $\varepsilon>0$ existe $\delta>0$ tal que

$$
\sum_{i=1}^{n}\left\|f\left(b_{i}\right)-f\left(a_{i}\right)\right\|<\varepsilon
$$

quando $a_{i} \leq b_{i}$ e $\left\{\left[a_{i}, b_{i}\right)_{\mathbb{T}}\right\}_{i=1}^{n}$ são intervalos disjuntos satisfazendo

$$
\sum_{i=1}^{n}\left(b_{i}-a_{i}\right)<\delta .
$$


Definição 2.2. Diz-se que a função $f: \mathbb{T} \rightarrow \mathbb{R}^{n}$ é um arco se $f$ é absolutamente contínua. Denotaremos o conjunto de todos os arcos com dominio $\mathbb{T}$ e contradominio $\mathbb{R}^{n}$ por $A C\left(\mathbb{T}, \mathbb{R}^{n}\right)$.

Teorema $2.1([2])$. Uma função $f: \mathbb{T} \rightarrow \mathbb{R}^{n}$ é absolutamente contínua se, e somente se, as seguintes condições são válidas:

(i) $\Delta$-q.t.p. $t \in[a, b)_{\mathbb{T}}$ a função $f$ é $\Delta$-diferenciável e $f^{\Delta} \in L_{1}\left([a, b)_{\mathbb{T}}, \mathbb{R}^{n}\right)$;

(ii) para cada $t \in \mathbb{T}$ tem-se

$$
f(t)=f(a)+\int_{[a, t)_{\mathbb{T}}} f^{\Delta}(s) \Delta s
$$

\subsection{Mensurabilidade de multifunções}

Seja $(\Omega, \mathcal{F})$ um espaço mensurável. Uma multifunção $\Gamma: \Omega \rightsquigarrow \mathbb{R}^{n}$ é uma aplicação que aplica pontos $x \in \Omega$ em subconjuntos $\Gamma(x)$ de $\mathbb{R}^{n}$. Uma multifunção $\Gamma: \Omega \rightsquigarrow \mathbb{R}^{n}$ é $\mathcal{F}$-mensurável quando o conjunto

$$
\Gamma^{-1}(V)=\{x \in \Omega: \Gamma(x) \cap V \neq \emptyset\}
$$

é $\mathcal{F}$-mensurável para cada conjunto compacto $V \subset \mathbb{R}^{n}$.

Uma função $\gamma: \Omega \rightarrow \mathbb{R}^{n}$ é uma seleção da multifunção $\Gamma$ quando $\gamma(x) \in \Gamma(x)$ para todo $x \in \Omega$.

Uma multifunção $\Gamma$ será fechada ou não-vazia quando sua imagem $\Gamma(x)$ for fechada ou nãovazia para cada ponto $x \in \Omega$.

Teorema $2.2([4])$. Tome um espaço mensurável $(\Omega, \mathcal{F})$ e uma multifunção $\Gamma: \Omega \rightsquigarrow \mathbb{R}^{m}$ nãovazia e fechada. Se $\Gamma$ é $\mathcal{F}$-mensurável então $\Gamma$ admite uma seleção mensurável.

Teorema $2.3([4])$. Seja $\Gamma:[a, b] \rightsquigarrow \mathbb{R}^{n}$ uma multifunção fechada e $E:=\{t \in[a, b]: \Gamma(t) \neq \emptyset\}$. Então as seguintes afirmações são equivalentes:

(i) a multifunção $\Gamma$ é $\mathcal{L}$-mensurável.

(ii) o conjunto $\operatorname{Gr} \Gamma=\{(t, v): v \in \Gamma(t)\}$ é $\mathcal{L} \times \mathcal{B}^{n}$-mensurável.

(iii) o conjunto $E$ é $\mathcal{L}$-mensurável e existe uma sequência de funções $\mathcal{L}$-mensuráveis $\gamma_{i}: E \rightarrow$ $\mathbb{R}^{n}$ tal que

$$
\Gamma(t)=\overline{\left\{\gamma_{i}(t): i=1,2, \ldots\right\}}
$$

para cada $t \in E$.

Corolário 2.2. Sejam $\Gamma_{1}, \Gamma_{2}:[a, b] \rightsquigarrow \mathbb{R}^{n}$ multifunções fechadas. Se $\Gamma_{1}$ e $\Gamma_{2}$ são $\mathcal{L}$-mensuráveis então a multifunção $\Gamma:[a, b] \rightsquigarrow \mathbb{R}^{n}$ dada por

$$
\Gamma(t)=\Gamma_{1}(t) \cap \Gamma_{2}(t)
$$

é $\mathcal{L}$-mensurável.

\section{Teorema de seleção de Filippov em escalas temporais}

Considere o sistema de controle de equações

$$
\left\{\begin{array}{l}
x^{\Delta}(t)=f(t, x(\sigma(t)), u(t)) \quad \Delta-\text { q.t.p. } t \in[a, b)_{\mathbb{T}} \\
u(t) \in U(t) \Delta-\text { q.t.p. } t \in[a, b)_{\mathbb{T}} .
\end{array}\right.
$$

Se um par de processo de controle $(x, u)$ satisfaz a restrição (1) então $x$ satisfaz a seguinte inclusão dinâmica

$$
x^{\Delta}(t) \in\{f(t, x(\sigma(t)), u): u \in U(t)\} \quad \Delta-\text { q.t.p. } t \in[a, b)_{\mathbb{T}} .
$$


No Teorema 3.1 provaremos a afirmação inversa. Isto é, se um $\operatorname{arco} x \in A C\left(\mathbb{T}, \mathbb{R}^{n}\right)$ satisfaz (2) então existe uma seleção $\Delta$-mensurável $u$ de $U$ tal que o processo $(x, u)$ satisfaz $(1)$.

Tome uma função $\phi: \mathbb{T} \times \mathbb{R}^{m} \rightarrow \mathbb{R}^{n}$. Dizemos que $\phi$ é uma $\Delta$-função de Carathéodory se satisfizer as seguintes propriedades:

(i) para cada $t \in \mathbb{T}$ a função $x \mapsto \phi(t, x)$ é contínua.

(ii) para cada $x \in \mathbb{R}^{m}$ a função $t \mapsto \phi(t, x)$ é $\Delta$-mensurável.

Lema 3.1 ([8]). Seja $\phi:[a, b] \times \mathbb{R}^{m} \rightarrow \mathbb{R}^{n}$ uma $\mathcal{L}$-função de Carathéodory. Então $\phi$ é $\mathcal{L} \times \mathcal{B}^{m}$ mensurável.

Proposição 3.1 ([12]). Seja $\phi: \mathbb{T} \times \mathbb{R}^{m} \rightarrow \mathbb{R}^{n}$ uma função $\Delta \times \mathcal{B}^{m}$-mensurável e y : T $\rightarrow \mathbb{R}^{m}$ uma função $\Delta$-mensurável. Então a função $g(t)=\phi(t, y(t))$ é $\Delta$-mensurável.

Lema $3.2([12])$. Seja $\phi: \mathbb{T} \times \mathbb{R}^{m} \rightarrow \mathbb{R}^{n}$ uma $\Delta$-função de Carathéodory. Então a função $\tilde{\phi}:[a, b] \times \mathbb{R}^{m} \rightarrow \mathbb{R}^{n}$ definida como

$$
\tilde{\phi}(t, u)=\left\{\begin{array}{l}
\phi(t, u), t \in \mathbb{T} \\
\phi\left(t_{i}, u\right), \quad t \in\left(t_{i}, \sigma\left(t_{i}\right)\right) \text { para algum } i \in I
\end{array}\right.
$$

é uma $\mathcal{L}$-função de Carathéodory.

Lema $3.3([12])$. Seja $U: \mathbb{T} \rightsquigarrow \mathbb{R}^{m}$ uma multifunção $\Delta$-mensurável. Então a multifunção $\tilde{U}:[a, b] \rightsquigarrow \mathbb{R}^{m}$ dada por

$$
\tilde{U}(t)=\left\{\begin{array}{l}
U(t), \quad t \in \mathbb{T} \\
U\left(t_{i}\right), \quad t \in\left(t_{i}, \sigma\left(t_{i}\right)\right) \text { para algum } i \in I
\end{array}\right.
$$

é $\mathcal{L}$-mensurável.

Teorema 3.1. Seja $U: \mathbb{T} \rightsquigarrow \mathbb{R}^{m}$ uma multifunção não-vazia, fechada e $\Delta$-mensurável. Considere uma função $f: \mathbb{T} \times \mathbb{R}^{n} \times \mathbb{R}^{m} \rightarrow \mathbb{R}^{n}$ contínua em $(x, u)$ para cada $t$ fixado, e $\Delta$-mensurável em t para cada $(x, u)$ fixado.

Se $x \in A C\left(\mathbb{T}, \mathbb{R}^{n}\right)$ satisfaz (2) então existe uma seleção $\Delta$-mensurável u de $U$ tal que o processo $(x, u)$ satisfaz (1).

Demonstração. Seja $u \in \mathbb{R}^{m}$ fixado arbitrariamente. Então a função $\varphi: \mathbb{T} \times \mathbb{R}^{n} \rightarrow \mathbb{R}^{n}$ dada por

$$
\varphi(t, x)=f(t, x, u)
$$

é uma $\Delta$-função de Carathéodory.

Do Lema 3.2 a função $\tilde{\varphi}:[a, b] \times \mathbb{R}^{n} \rightarrow \mathbb{R}^{n}$ é uma $\mathcal{L}$-função de Carathéodory. Logo, do Lema 3.1 a função $\tilde{\varphi}$ é $\mathcal{L} \times \mathcal{B}^{n}$-mensurável.

Sendo $y=x \circ \sigma$ uma função $\Delta$-mensurável, da Proposição 2.1 a função $\tilde{y}:[a, b] \rightarrow \mathbb{R}^{n}$ é $\mathcal{L}$-mensurável. Assim, da Proposição 3.1 a função

$$
t \mapsto \tilde{\varphi}(t, \tilde{y}(t))
$$

é $\mathcal{L}$-mensurável.

Defina a função $g: \mathbb{T} \rightarrow \mathbb{R}^{n}$ como

$$
g(t)=f(t, x(\sigma(t)), u) .
$$

Temos que $\tilde{\varphi}(t, \tilde{y}(t))=\tilde{g}(t)$ para cada $t \in[a, b]$. Assim, a função

$$
t \mapsto \tilde{g}(t)
$$

é $\mathcal{L}$-mensurável. 
Seja $m:[a, b] \times \mathbb{R}^{m} \rightarrow \mathbb{R}^{n}$ definida como

$$
m(t, u)=\left\{\begin{array}{l}
f(t, x(\sigma(t)), u), t \in \mathbb{T} \\
f\left(t_{i}, x\left(\sigma\left(t_{i}\right)\right), u\right), \quad t \in\left(t_{i}, \sigma\left(t_{i}\right)\right) \text { para algum } i \in I .
\end{array}\right.
$$

Para cada $u \in \mathbb{R}^{m}$ já provamos que a função

$$
t \mapsto m(t, u)
$$

é $\mathcal{L}$-mensurável. Além disso, para cada $t \in[a, b]$ a função

$$
u \mapsto m(t, u)
$$

é contínua.

Como $x$ é um arco, a função $h: \mathbb{T} \rightarrow \mathbb{R}^{n}$ dada por

$$
h(s)=x^{\Delta}(s)
$$

é $\Delta$-mensurável. Logo, a função $\tilde{h}:[a, b] \rightarrow \mathbb{R}^{n}$ é $\mathcal{L}$-mensurável. Então a função $\phi:[a, b] \times \mathbb{R}^{m} \rightarrow$ $\mathbb{R}^{n}$ definida por

$$
\phi(t, u)=m(t, u)-\tilde{h}(t)
$$

é uma $\mathcal{L}$-função de Carathéodory.

Seja $\Phi:[a, b] \rightsquigarrow \mathbb{R}^{m}$ a multifunção definida como

$$
\Phi(t)=\left\{u \in \mathbb{R}^{m}: \phi(t, u)=0\right\}
$$

para cada $t \in[a, b]$.

Para cada $t \in[a, b]$ a função

$$
u \mapsto \phi(t, u)
$$

é contínua. Assim, se $\Phi(t) \neq \emptyset$ então $\Phi(t)$ é um conjunto fechado.

Como $\phi$ é $\mathcal{L} \times \mathcal{B}^{m}$-mensurável então o conjunto

$$
\begin{aligned}
G r \Phi & =\left\{(t, u) \in[a, b] \times \mathbb{R}^{m}: u \in \Phi(t)\right\} \\
& =\left\{(t, u) \in[a, b] \times \mathbb{R}^{m}: \phi(t, u)=0\right\}=\phi^{-1}(\{0\})
\end{aligned}
$$

é $\mathcal{L} \times \mathcal{B}^{m}$-mensurável. Do Teorema 2.3 a multifunção $\Phi$ é $\mathcal{L}$-mensurável.

Sendo $\tilde{U}:[a, b] \rightsquigarrow \mathbb{R}^{m}$ uma multifunção $\mathcal{L}$-mensurável, do Corolário 2.2 a multifunção $\Gamma:[a, b] \rightsquigarrow \mathbb{R}^{m}$ dada por

$$
\Gamma(t)=\tilde{U}(t) \cap \Phi(t)
$$

é $\mathcal{L}$-mensurável.

Sejam os conjuntos

$$
\mathcal{K}=\left\{t \in[a, b)_{\mathbb{T}}:(2) \text { vale }\right\}
$$

e

$$
\mathcal{W}=\left\{t \in[a, b)_{\mathbb{T}}: U(t) \cap \Phi(t) \neq \emptyset\right\} .
$$

Se $t \in \mathcal{K}$, existe $u_{t} \in U(t)$ tal que

$$
\begin{aligned}
0 & =f\left(t, x(\sigma(t)), u_{t}\right)-x^{\Delta}(t) \\
& =f\left(t, x(\sigma(t)), u_{t}\right)-h(t)=m\left(t, u_{t}\right)-\tilde{h}(t)=\phi\left(t, u_{t}\right)
\end{aligned}
$$

e então $u_{t} \in U(t) \cap \Phi(t)$. Assim, $t \in \mathcal{W}$ e portanto $\mathcal{K} \subset \mathcal{W}$.

Entretanto, se $t \in \mathcal{W}$ existe $u_{t} \in U(t)$ satisfazendo

$$
\begin{aligned}
0 & =\phi\left(t, u_{t}\right)=m\left(t, u_{t}\right)-\tilde{h}(t) \\
& =f\left(t, x(\sigma(t)), u_{t}\right)-h(t)=f\left(t, x(\sigma(t)), u_{t}\right)-x^{\Delta}(t)
\end{aligned}
$$


isto é,

$$
x^{\Delta}(t)=f\left(t, x(\sigma(t)), u_{t}\right)
$$

e então $t \in \mathcal{K}$. Concluímos que $\mathcal{W}=\mathcal{K}$.

Assim, o conjunto $E$ dado por

$$
E=\left\{t \in[a, b)_{\mathbb{T}}: U(t) \cap \Phi(t)=\emptyset\right\}
$$

satisfaz

$$
E=[a, b)_{\mathbb{T}} \backslash \mathcal{W}=[a, b)_{\mathbb{T}} \backslash \mathcal{K}
$$

Portanto

$$
\mu_{\Delta}(E)=\mu_{\Delta}\left([a, b)_{\mathbb{T}} \backslash \mathcal{K}\right)=0 .
$$

Se a multifunção $\Lambda: \mathbb{T} \rightsquigarrow \mathbb{R}^{m}$ for definida como

$$
\Lambda(t)=\left\{\begin{array}{l}
U(t), t \in E \cup\{b\} \\
U(t) \cap \Phi(t)=\Gamma(t), \quad t \in \mathbb{T} \backslash\{E \cup\{b\}\}
\end{array}\right.
$$

então $\Lambda$ é não-vazia e fechada.

Se $V \subset \mathbb{R}^{m}$ é um conjunto compacto, segue que

$$
\begin{aligned}
\Lambda^{-1}(V) & =\{t \in \mathbb{T}: \Lambda(t) \cap V \neq \emptyset\} \\
& =\left[U^{-1}(V) \cap(E \cup\{b\})\right] \bigcup\left[\Gamma^{-1}(V) \cap(\mathbb{T} \backslash\{E \cup\{b\}\})\right] .
\end{aligned}
$$

Como $U$ é $\Delta$-mensurável, $\Gamma$ é $\mathcal{L}$-mensurável e os conjuntos $E \cup\{b\}$ e $\mathbb{T} \backslash\{E \cup\{b\}\}$ são $\Delta$ mensuráveis, usando o Lema 2.2 concluímos que $\Lambda^{-1}(V) \in \Delta$. Portanto $\Lambda$ é $\Delta$-mensurável.

Do Teorema 2.2 a multifunção $\Lambda$ admite uma seleção $\Delta$-mensurável $u: \mathbb{T} \rightarrow \mathbb{R}^{m}$. Assim, $u(t) \in U(t)$ para todo $t \in \mathbb{T}$ e então $u$ também é uma seleção de $U$.

Se $t \in[a, b)_{\mathbb{T}} \backslash E$ segue que $u(t) \in U(t) \cap \Phi(t)$. Logo,

$$
\begin{aligned}
0 & =\phi(t, u(t))=m(t, u(t))-\tilde{h}(t)=f(t, x(\sigma(t)), u(t))-h(t) \\
& =f(t, x(\sigma(t)), u(t))-x^{\Delta}(t)
\end{aligned}
$$

isto é,

$$
x^{\Delta}(t)=f(t, x(\sigma(t)), u(t))
$$

Portanto

$$
x^{\Delta}(t)=f(t, x(\sigma(t)), u(t)) \quad \Delta-q . t . p . \quad t \in[a, b)_{\mathbb{T}}
$$

já que $\mu_{\Delta}(E)=0$. Então $(x, u)$ satisfaz o sistema de controle de equações (1).

\section{Conclusões}

Esse trabalho contribuiu para a teoria de escalas temporais. Mais especificamente, obtém uma extensão do teorema de seleção de Filippov para escalas temporais. Considerando a metodologia para o estudo da existência de soluções para problemas de controle ótimo [5], [10] e [12], acreditamos que esse trabalho também possa contribuir para o estudo da existência de soluções para problemas de controle ótimo em escalas temporais. 


\section{Referências}

[1] R.G. Bartle, "The Elements of Integration and Lebesgue Measure", John Wiley and Sons, New York, 1995.

[2] A. Cabada, D.R. Vivero, Criterions for absolute continuity on time scales, Journal of Difference Equations and Applications, 11 (2005) 1013-1028.

[3] A. Cabada, D.R. Vivero, Expression of the Lebesgue $\Delta$-integral on time scales as a usual Lebesgue integral; application to the calculus of $\Delta$-antiderivatives, Mathematical and Computer Modelling, 43 (2006) 194-207.

[4] C. Castaing, M. Valadier, "Convex Analysis and Measurable Multifunctions", Vol. 580, Springer Lecture Notes in Mathematics, Berlin, 1977.

[5] A.F. Filippov, On certain questions in the theory of Optimal Control, SIAM J. Control Optimization, 1 (1962) 76-84.

[6] G.S. Guseinov, Integration on time scales, Journal of Mathematical Analysis and Applications, 285 (2003) 107-127.

[7] R. Hilscher, V. Zeidan, Weak maximum principle and accessory problem for control problems on time scales, Nonlinear Analysis, 70 (2009) 3209-3226.

[8] P.D. Loewen, "Optimal Control via Nonsmooth Analysis", CRM Proceedings Lecture Notes, Vol.2, American Mathematical Society, Providence, RI, 1993.

[9] H.L. Royden, "Real Analysis", Collier-Macmillan Limited, London, 1968.

[10] E. Roxin, The existence of optimal controls, The Michigan Mathematical Journal, 9 (1962) 109-119.

[11] W. Rudin, "Real and Complex Analysis", third edition, McGraw-Hill Book Company, New York, 1987.

[12] I.L.D. Santos, G.N. Silva, Filippov's selection theorem and the existence of solutions for optimal control problems in time scales, Computational and Applied Mathematics, 33 (2013) 223-241.

[13] I.L.D. Santos, G.N. Silva, Uma classe de problemas de controle ótimo em escalas temporais, Anais do SBAI/DINCON 2013, artigo aceito para publicação. 